\title{
Orientational Ordering of Polymers on a Fluctuating Flexible Surface
}

\author{
R.Podgornik* \\ Laboratory of Structural Biology \\ Division of Computer Research and Technology \\ National Institutes of Health, Bethesda, MD 20892
}

\begin{abstract}
Arguments are presented to the effect that embedding semi-flexible (wormlike) ideal polymers into a fluctuating, flexible surface leads to an effective attractive orientational interaction between polymer segments that precipitates an orientational ordering transition of the polymer chains on the surface even in the case of otherwise ideal (noninteracting) chains. The orientational interaction is analogous to the (zero order) Casimir force and is due to the energy change in surface conformational fluctuations in the presence of embedded semi-flexible chains.

August 15, 2018
\end{abstract}

${ }^{*}$ On leave from J.Stefan Institute, P.O.B. 100, Ljubljana, Slovenia. 


\section{Introduction}

Polymers or polymer-like structures confined to lye in an embedding surface can appear in the context of different membrane and/or interfacial phenomena. Partially polymerized membranes of unsaturated amphiphiles can sometimes make long trains of joined monomers that behave as surface - embedded polymer chains [1]. Hydrophobic polymers in aqueous solutions can become embedded or multiply attached to hydrocarbon interior of membranes [1], while certain polymers can also form monomolecular films on the water-air interface [2]. It has been observed that embedded polymers in the case of partially polymerized membranes can promote wrinkling transition in membranes [3] while multiply anchored polymers give rise to bulging and budding of membranes [4. In the case of interface polymer films [2] it has been shown that they make segregated domains even at very low (submonolayer) concentrations where one would expect the polymers to be evenly distributed over the surface.

This plethora of new phenomena that appear closely connected with the fact that polymers are not only adsorbed but actually embedded into a supporting surface have recently become a focus of several theoretical studies [5]. In this contribution we try to find out what is the influence of finite temperature, which in general leads to shape fluctuations in flexible (membrane) surfaces or interfaces, on the properties of embedded polymer chains. In the framework of a semiflexible polymer chain model we show that finite temperature leads to membrane fluctuation generated effective orienting forces between different polymer segments. This interaction formally resembles the usual nematic interaction used in various models of the nematic ordering of long flexible chains [12] and is thus not surprising that it leads to an orientational ordering transition.

The interesting point is that this transition is driven by shape fluctuations of the supporting surface into which the polymer is embedded.

The problem we address here is related to the recent investigations of fluctuation-induced forces between manifolds immersed in a correlated fluid [6]. The existence of surface fluctuations promoted attraction between different segments of the embedded polymer chain can also be related to the zero - order Casimir forces (Keesom forces) between beads attached to flexible membranes [9]. Other related problems include adsorption of polymers on soft, fluctuating surfaces [7] or the collapse of polymers on fluctuating surfaces [8].

The outline of the paper is as follows: I shall first derive the partition 
function of a surface embedded semiflexible polymer chain at zero temperature. Then I shall relax the zero temperature constraint and derive the effective partition function for a polymer, after the supporting surface degrees of freedom have been integrated out. At last an approximate solution for this effective partition function will be given and discussed.

\section{Analysis}

\subsection{Semiflexible polymer chain embedded in a surface}

I use a semiflexible (worm - like chain) model to describe the polymer chain. Though the theory presented below is formulated for a single long polymer chain, it can be trivially generalized to a many - chain system by assuming that the variable $n$ has a continuous part $N_{\alpha}$, describing a single chain, and a discrete part describing different chains $\alpha$.

I investigate the statistical properties of a semiflexible polymer chain embedded in a fluctuating surface. Let us assume that the n-th bead of the polymer has coordinates $\mathbf{r}(n)=(x(n), y(n), z(n))$ and that the quenched profile of the embedding surface can be written in a Monge parametrization $z=\zeta(x, y)=\zeta(\boldsymbol{\rho})$, where I introduced the two dimensional vector $\boldsymbol{\rho}=(x, y)$. The embedding ansatz for the polymer can thus be written in a form

$$
z(n)=\zeta(\boldsymbol{\rho}(n)) .
$$

I also assume that the polymers can be described in terms of the semiflexible chain model, where the configurational energy is given as

$$
\beta \mathcal{H}_{0}(\mathbf{r}(n))=\frac{1}{2} \beta \epsilon \int_{0}^{N}\left(\frac{d^{2} \mathbf{r}(n)}{d n^{2}}\right)^{2} d n=\frac{1}{2} \beta \epsilon \int_{0}^{N}(\ddot{\mathbf{r}}(n))^{2} d n
$$

where $\beta \epsilon=\frac{1}{\ell^{2}}$, with $\ell$ being the persistence length and $N$ is the number of monomers. For a differentiable curve the tangent $\dot{\mathbf{r}}(n)$ is a unit vector, $\dot{\mathbf{r}}(n)^{2}=1$. The partition function of a semiflexible chain embedded in the surface $\zeta(x, y)$ can thus be written in a succinct form

$\Xi(\zeta(\boldsymbol{\rho}(n)))=\int \ldots \int \mathcal{D} \mathbf{r}(n) \prod_{n} \delta(z(n)-\zeta(\boldsymbol{\rho}(n))) \prod_{n} \delta\left(\dot{\mathbf{r}}(n)^{2}-1\right) \times e^{-\beta \mathcal{H}_{0}(\mathbf{r}(n))}$.

The explicit incorporation of the constraint that the local tangent has to be a unit vector permits us to perform the functional integration in Eq.3 over an unconstrained set of $\mathbf{r}(n)$. 
By integrating out the $z(n)$ variables and introducing an integral representation for the delta function I obtain the partition function in the form

$$
\Xi(\zeta(\boldsymbol{\rho}(n)))=\int \ldots \int \mathcal{D} \boldsymbol{\rho}(n) \mathcal{D} \lambda(n) e^{-\beta \mathcal{H}(\boldsymbol{\rho}(n), \zeta(\rho(n)))}
$$

where the effective configurational Hamiltonian reads

$$
\beta \mathcal{H}(\boldsymbol{\rho}(n), \zeta(\boldsymbol{\rho}(n)))=\frac{1}{2} \beta \epsilon \int_{0}^{N}\left(\ddot{\boldsymbol{\rho}}(n)^{2}+\ddot{\zeta}(\boldsymbol{\rho}(n))^{2}\right) d n+\imath \int_{0}^{N} \lambda(n)\left(\dot{\boldsymbol{\rho}}(n)^{2}+\dot{\zeta}(\boldsymbol{\rho}(n))^{2}\right) d n
$$

It is at this point where a first major approximation is necessary in order to proceed. Instead of enforcing the condition $\dot{\mathbf{r}}(n)^{2}=1$ locally at each point along the polymer chain, we presume that it is only satisfied globally [10] i.e. on the average

$$
\left\langle\dot{\mathbf{r}}(n)^{2}\right\rangle=\left\langle\dot{\boldsymbol{\rho}}(n)^{2}+\dot{\zeta}(\boldsymbol{\rho}(n))^{2}\right\rangle=1,
$$

where the average is over all polymer configurations. Thus the magnitude of the tangent can fluctuate locally but on the average, over the entire polymer chain, it is fixed. Replacement of the local continuity constraint with a global one Eq. 6 can also expressed as $\lambda(n) \longrightarrow \lambda=$ const.. Thus one remains (after making at the same time also the transformation $\imath \lambda \longrightarrow \lambda$ ) with the following form of the partition function

$$
\Xi(\zeta(\boldsymbol{\rho}(n)))=\int \ldots \int \mathcal{D} \boldsymbol{\rho}(n) \exp (-\beta \mathcal{H}(\boldsymbol{\rho}(n))),
$$

where some irrelevant constant terms have been omitted. Also

$$
\begin{aligned}
\beta \mathcal{H}(\boldsymbol{\rho}(n)) & =\frac{1}{2} \beta \epsilon \int_{0}^{N}\left(\ddot{\boldsymbol{\rho}}(n)^{2}+\ddot{\zeta}(n)^{2}\right) d n+ \\
& +\lambda \int_{0}^{N}\left(\dot{\boldsymbol{\rho}}(n)^{2}+\dot{\zeta}(n)^{2}\right) d n \\
& \cong \frac{1}{2} \beta \epsilon \int_{0}^{N}\left(\ddot{\boldsymbol{\rho}}(n)^{2}+\frac{\partial \zeta(\boldsymbol{\rho})}{\partial \rho_{i}} \frac{\partial \zeta(\boldsymbol{\rho})}{\partial \rho_{k}} \ddot{\boldsymbol{\rho}}_{i}(n) \ddot{\boldsymbol{\rho}}_{k}(n)\right) d n+ \\
& +\lambda \int_{0}^{N}\left(\dot{\boldsymbol{\rho}}(n)^{2}+\frac{\partial \zeta(\boldsymbol{\rho})}{\partial \rho_{i}} \frac{\partial \zeta(\boldsymbol{\rho})}{\partial \rho_{k}} \dot{\boldsymbol{\rho}}_{i}(n) \dot{\boldsymbol{\rho}}_{k}(n)\right) d n+\ldots
\end{aligned}
$$

where we have limited ourselves to the terms of the second order in the derivatives of $\boldsymbol{\rho}(n)$.

\subsection{Conformational fluctuations of the embedding sur- face}

Going now to a non-zero temperature one has to take into account that the embedding surface is allowed to fluctuate, taking the polymer with it on its 
conformational wanderings. The unrestrained partition function in this case is

$$
\Xi(N)=\int \ldots \int \mathcal{D} \boldsymbol{\rho}(n) \mathcal{D} \zeta(\boldsymbol{\rho}) \exp \left(-\beta \mathcal{H}_{T}(\zeta(\boldsymbol{\rho}), \boldsymbol{\rho}(n))\right)
$$

where after introducing the Fourier transform $\zeta(\boldsymbol{\rho})=\sum_{\mathbf{Q}} \zeta(\mathbf{Q}) e^{\imath \mathbf{Q} \boldsymbol{\rho}}$

$$
\begin{aligned}
\beta \mathcal{H}_{T}(\zeta(\boldsymbol{\rho}), \boldsymbol{\rho}(n))= & \frac{1}{2} \beta \epsilon \int_{0}^{N} \ddot{\boldsymbol{\rho}}(n)^{2} d n+\lambda \int_{0}^{N} \dot{\boldsymbol{\rho}}(n)^{2} d n+ \\
& \frac{1}{2} \sum_{\mathbf{Q}, \mathbf{Q}^{\prime}} \zeta(\mathbf{Q}) \mathcal{D}\left(\mathbf{Q}, \mathbf{Q}^{\prime}\right) \zeta\left(-\mathbf{Q}^{\prime}\right)+\frac{1}{2} \beta \sum_{\mathbf{Q}} V(\zeta(\mathbf{Q}))
\end{aligned}
$$

where

$$
\begin{aligned}
\mathcal{D}\left(\mathbf{Q}, \mathbf{Q}^{\prime} ; \boldsymbol{\rho}(n)\right) & =\beta \epsilon \int_{0}^{N} d n(\mathbf{Q} \ddot{\boldsymbol{\rho}}(n))\left(\mathbf{Q}^{\prime} \ddot{\boldsymbol{\rho}}(n)\right) e^{\imath\left(\mathbf{Q}-\mathbf{Q}^{\prime}\right) \boldsymbol{\rho}(n)}+ \\
& +2 \lambda \int_{0}^{N} d n(\mathbf{Q} \dot{\boldsymbol{\rho}}(n))\left(\mathbf{Q}^{\prime} \dot{\boldsymbol{\rho}}(n)\right) e^{\imath\left(\mathbf{Q}-\mathbf{Q}^{\prime}\right) \boldsymbol{\rho}(n)}
\end{aligned}
$$

$V(\zeta(\mathbf{Q}))$ is the intrinsic deformation energy of the surface describing the energetics of the surface in the absence of embedded polymers, I assume it to be of the form

$$
V(\zeta(\mathbf{Q}))=V(Q)|\zeta(\mathbf{Q})|^{2}
$$

With this ansatz one can integrate out the $\zeta(\boldsymbol{\rho})$ degrees of freedom obtaining

$$
\begin{aligned}
\Xi(\boldsymbol{\rho}(n)) & =\int \ldots \int \mathcal{D} \zeta(\boldsymbol{\rho}) \Xi(\zeta(\boldsymbol{\rho}), \boldsymbol{\rho}(n))= \\
& =\int \ldots \int \mathcal{D} \boldsymbol{\rho}(n) \times \exp \left(-\frac{1}{2} \beta \epsilon \int_{0}^{N} \ddot{\boldsymbol{\rho}}(n)^{2} d n-\lambda \int_{0}^{N} \dot{\boldsymbol{\rho}}(n)^{2} d n-\frac{1}{2} \operatorname{Tr} \ln \mathcal{G}\left(\mathbf{Q}, \mathbf{Q}^{\prime} ; \boldsymbol{\rho}(n)\right)\right)
\end{aligned}
$$

with

$$
\mathcal{G}\left(\mathbf{Q}, \mathbf{Q}^{\prime} ; \boldsymbol{\rho}(n)\right)=(\beta V(Q)) \delta\left(\mathbf{Q}-\mathbf{Q}^{\prime}\right)+\mathcal{D}\left(\mathbf{Q}, \mathbf{Q}^{\prime} ; \boldsymbol{\rho}(n)\right) .
$$

At this point I use the standard identity relating $\operatorname{Tr} \ln$ of an operator with a coupling integral of its resolvent

$$
\operatorname{Tr} \ln \mathcal{G}\left(\mathbf{Q}, \mathbf{Q}^{\prime} ; \boldsymbol{\rho}(n)\right)=\operatorname{Tr} \int_{0}^{1} d \mu \mathcal{R}_{\mu}\left(\mathbf{Q}, \mathbf{Q}^{\prime} ; \boldsymbol{\rho}(n)\right)
$$

where the resolvent $\mathcal{R}_{\mu}\left(\mathbf{Q}, \mathbf{Q}^{\prime} ; \boldsymbol{\rho}(n)\right)$ is defined as

$$
\mathcal{R}_{\mu}\left(\mathbf{Q}, \mathbf{Q}^{\prime} ; \boldsymbol{\rho}(n)\right)=\mathcal{D}\left(\mathbf{Q}, \mathbf{Q}^{\prime} ; \boldsymbol{\rho}(n)\right)(\beta V(Q))^{-1} \times\left(\mathcal{I}+\mu \mathcal{D}\left(\mathbf{Q}, \mathbf{Q}^{\prime} ; \boldsymbol{\rho}(n)\right)(\beta V(Q))^{-1}\right)^{-1}
$$

with $\mathcal{I}$ being the identity operator in $Q$ space. I have omitted a term that does not depend on the coordinates $\boldsymbol{\rho}(n)$ from Eq.15. Evaluating the resolvent to the first order in $\mu$ I obtain

$\operatorname{Tr} \ln \mathcal{G}\left(\mathbf{Q}, \mathbf{Q}^{\prime} ; \boldsymbol{\rho}(n)\right) \cong \operatorname{Tr} \mathcal{D}\left(\mathbf{Q}, \mathbf{Q}^{\prime} ; \boldsymbol{\rho}(n)\right)(\beta V(Q))^{-1}-\frac{1}{2} \operatorname{Tr}\left(\mathcal{D}\left(\mathbf{Q}, \mathbf{Q}^{\prime} ; \boldsymbol{\rho}(n)\right)(\beta V(Q))^{-1}\right)^{2}+\ldots$ 
What one remains with at the end is an effective interaction between polymer segments as if they would be confined to a planar surface. It has the form

$$
\begin{aligned}
& \frac{1}{2} \operatorname{Tr} \ln \mathcal{G}\left(\mathbf{Q}, \mathbf{Q}^{\prime} ; \boldsymbol{\rho}(n)\right) \cong \frac{\beta \epsilon}{2} \int_{0}^{N} d n \ddot{\boldsymbol{\rho}}_{i}(n) \ddot{\boldsymbol{\rho}}_{k}(n) \sum_{\mathbf{Q}} \frac{\mathbf{Q}_{i} \mathbf{Q}_{k}}{(\beta V(Q))}+ \\
&+\lambda \int_{0}^{N} d n \dot{\boldsymbol{\rho}}_{i}(n) \dot{\boldsymbol{\rho}}_{k}(n) \frac{\mathbf{Q}_{i} \mathbf{Q}_{k}}{(\beta V(Q))}- \\
&-\frac{(2 \lambda)^{2}}{4} \int_{0}^{N} \int_{0}^{N} d n d n^{\prime} \dot{\boldsymbol{\rho}}_{i}(n) \dot{\boldsymbol{\rho}}_{k}(n) \dot{\boldsymbol{\rho}}_{l}\left(n^{\prime}\right) \dot{\boldsymbol{\rho}}_{m}\left(n^{\prime}\right) \sum_{\mathbf{Q}} \frac{\mathbf{Q}_{i} \mathbf{Q}_{k}}{(\beta V(Q))} e^{\imath \mathbf{Q}\left(\boldsymbol{\rho}(n)-\boldsymbol{\rho}\left(n^{\prime}\right)\right)} \sum_{\mathbf{Q}^{\prime}} \frac{\mathbf{Q}_{l}^{\prime} \mathbf{Q}_{m}^{\prime}}{\left(\beta V\left(Q^{\prime}\right)\right)} e^{-\imath \mathbf{Q}^{\prime}\left(\boldsymbol{\rho}(n)-\boldsymbol{\rho}\left(n^{\prime}\right)\right)}+\ldots
\end{aligned}
$$

I have limited myself to second order terms in the derivatives of $\boldsymbol{\rho}(n)$ at any $n$ (self - energy term) and to fourth order terms for two different values of $n$ (interaction term).

Introducing now the orientational correlation function between the directions of the surface normals $\mathbf{n}(\boldsymbol{\rho})$ in the absence of polymer chains and for small surface deformations as

$$
\left\langle\mathbf{n}_{i}(\boldsymbol{\rho}) \mathbf{n}_{k}\left(\boldsymbol{\rho}^{\prime}\right)\right\rangle \cong\left\langle\frac{\partial \zeta(\boldsymbol{\rho})}{\partial \boldsymbol{\rho}_{i}} \frac{\partial \zeta\left(\boldsymbol{\rho}^{\prime}\right)}{\partial \boldsymbol{\rho}_{k}^{\prime}}\right\rangle=-\sum_{\mathbf{Q}, \mathbf{Q}^{\prime}} \mathbf{Q}_{i} \mathbf{Q}_{k}^{\prime}\left\langle\zeta(\mathbf{Q}) \zeta\left(\mathbf{Q}^{\prime}\right)\right\rangle e^{\imath\left(\mathbf{Q} \boldsymbol{\rho}+\mathbf{Q}^{\prime} \boldsymbol{\rho}^{\prime}\right)}
$$

where due to the harmonic ansatz for the configurational energy of the bare surface I have

$$
\left\langle\zeta(\mathbf{Q}) \zeta\left(\mathbf{Q}^{\prime}\right)\right\rangle=\frac{1}{2} \frac{k T}{V(Q)} \delta\left(\mathbf{Q}+\mathbf{Q}^{\prime}\right)
$$

thus

$$
\beta\left\langle\mathbf{n}_{i}(\boldsymbol{\rho}) \mathbf{n}_{k}\left(\boldsymbol{\rho}^{\prime}\right)\right\rangle=\frac{1}{2} \beta \mathcal{F}_{i k}\left(\boldsymbol{\rho}-\boldsymbol{\rho}^{\prime}\right)=\frac{1}{2} \sum_{\mathbf{Q}} \frac{\mathbf{Q}_{i} \mathbf{Q}_{k}}{V(Q)} e^{\imath \mathbf{Q}\left(\boldsymbol{\rho}-\boldsymbol{\rho}^{\prime}\right)} .
$$

The final expression for the partition function of a surface - embedded polymer chain at finite temperature is thus

$$
\Xi(N)=\int \ldots \int \mathcal{D} \boldsymbol{\rho}(n) e^{-\beta \mathcal{H}_{e f f}(\dot{\boldsymbol{\rho}}(n), \ddot{\boldsymbol{\rho}}(n))}
$$

with

$$
\begin{aligned}
\beta \mathcal{H}_{e f f}(\dot{\boldsymbol{\rho}}(n), \ddot{\boldsymbol{\rho}}(n)) & =\frac{1}{2} \beta \epsilon \int_{0}^{N} \ddot{\boldsymbol{\rho}}(n)^{2} d n+\lambda \int_{0}^{N} \dot{\boldsymbol{\rho}}(n)^{2} d n+ \\
& +\beta \epsilon \int_{0}^{N} d n \ddot{\boldsymbol{\rho}}_{i}(n) \ddot{\boldsymbol{\rho}}_{k}(n)\left\langle\mathbf{n}_{i}(0) \mathbf{n}_{k}(0)\right\rangle+ \\
& +2 \lambda \int_{0}^{N} d n \dot{\boldsymbol{\rho}}_{i}(n) \dot{\boldsymbol{\rho}}_{k}(n)\left\langle\mathbf{n}_{i}(0) \mathbf{n}_{k}(0)\right\rangle- \\
& -4 \lambda^{2} \int_{0}^{N} \int_{0}^{N} d n d n^{\prime} \dot{\boldsymbol{\rho}}_{i}(n) \dot{\boldsymbol{\rho}}_{k}(n) \dot{\boldsymbol{\rho}}_{l}\left(n^{\prime}\right) \dot{\boldsymbol{\rho}}_{m}\left(n^{\prime}\right)\left\langle\mathbf{n}_{i}(n) \mathbf{n}_{m}\left(n^{\prime}\right)\right\rangle\left\langle\mathbf{n}_{k}\left(n^{\prime}\right) \mathbf{n}_{l}(n)\right\rangle+\ldots
\end{aligned}
$$


Obviously the integration of the surface degrees of freedom leads to an effective attraction between the polymer segments, which is orientation dependent. This attraction has the same origin as the usual (zero order) Casimir force [14] and we could simply dubb it the nematic Casimir force, or maybe more appropriately the nematic Keesom force. The fluctuations of the embedding surface play in this case the role of vacuum fluctuations of the electromagnetic field [13].

\subsection{Effective embedded - polymer partition function and orientational ordering}

Introducing now the orientational tensor $\sigma_{i k}(\boldsymbol{\rho})$ as [1]

$$
\sigma_{i k}(\boldsymbol{\rho})=\int_{0}^{N} d n \dot{\boldsymbol{\rho}}_{i}(n) \dot{\boldsymbol{\rho}}_{k}(n) \delta(\boldsymbol{\rho}-\boldsymbol{\rho}(n))
$$

I can write the partition function alternatively as

$$
\Xi(N)=\int \ldots \int \mathcal{D} \boldsymbol{\rho}(n) \mathcal{D} \sigma_{i k}(\boldsymbol{\rho}) \delta\left(\int_{0}^{N} d n \dot{\boldsymbol{\rho}}_{i}(n) \dot{\boldsymbol{\rho}}_{k}(n) \delta(\boldsymbol{\rho}-\boldsymbol{\rho}(n))-\sigma_{i k}(\boldsymbol{\rho})\right) e^{-\beta \mathcal{H}_{e f f}\left(\dot{\boldsymbol{\rho}}(n), \ddot{\boldsymbol{\rho}}(n), \sigma_{i k}(\boldsymbol{\rho})\right)}
$$

where

$$
\begin{aligned}
\beta \mathcal{H}_{e f f}\left(\dot{\boldsymbol{\rho}}(n), \ddot{\boldsymbol{\rho}}(n), \sigma_{i k}(\boldsymbol{\rho})\right) & =\frac{1}{2} \beta \epsilon \int_{0}^{N} \ddot{\boldsymbol{\rho}}(n)^{2} d n+\lambda \int_{0}^{N} \dot{\boldsymbol{\rho}}(n)^{2} d n+ \\
& +\frac{1}{2} \beta \epsilon \int_{0}^{N} d n \ddot{\boldsymbol{\rho}}_{i}(n) \ddot{\boldsymbol{\rho}}_{k}(n) \mathcal{F}_{i k}(0)+ \\
& +\lambda \int d^{2} \boldsymbol{\rho} \sigma_{i k}(\boldsymbol{\rho}) \mathcal{F}_{i k}(0)- \\
& -\lambda^{2} \iint d^{2} \boldsymbol{\rho} d^{2} \boldsymbol{\rho}^{\prime} \sigma_{i k}(\boldsymbol{\rho}) \sigma_{l m}\left(\boldsymbol{\rho}^{\prime}\right) \mathcal{F}_{i m}\left(\boldsymbol{\rho}-\boldsymbol{\rho}^{\prime}\right) \mathcal{F}_{k l}\left(\boldsymbol{\rho}-\boldsymbol{\rho}^{\prime}\right)+\ldots
\end{aligned}
$$

In order to proceed from here one has to specify the form of $V(Q)$, valid for the bare surface. To get some general trends and estimates I shall limit myself to the following simple form of $\mathcal{F}_{i k}(\boldsymbol{\rho})$

$$
\mathcal{F}_{i k}(\boldsymbol{\rho})=\delta_{i k} F(|\boldsymbol{\rho}|) \text {. }
$$

Also writing the delta function in an integral representation I obtain the following form of the effective Hamiltonian

$$
\beta \hat{\mathcal{H}}\left(\dot{\boldsymbol{\rho}}(n), \ddot{\boldsymbol{\rho}}(n), \sigma_{i k}(\boldsymbol{\rho}), \psi_{i k}(\boldsymbol{\rho})\right)=\beta \mathcal{H}_{e f f}\left(\dot{\boldsymbol{\rho}}(n), \ddot{\boldsymbol{\rho}}(n), \sigma_{i k}(\boldsymbol{\rho})\right)+\imath \int_{0}^{N} d n \dot{\boldsymbol{\rho}}_{i}(n) \dot{\boldsymbol{\rho}}_{k}(n) \psi_{i k}(\boldsymbol{\rho}(n))-
$$




$$
\begin{aligned}
& -\imath \int d^{2} \boldsymbol{\rho} \psi_{i k}(\boldsymbol{\rho}) \sigma_{i k}(\boldsymbol{\rho})= \\
& =\frac{1}{2} \beta \epsilon(1+F(0)) \int_{0}^{N} \ddot{\boldsymbol{\rho}}(n)^{2} d n+\lambda \int_{0}^{N} \dot{\boldsymbol{\rho}}(n)^{2} d n+ \\
& +\quad(\lambda F(0)) \int d^{2} \boldsymbol{\rho} \operatorname{Tr}\left(\sigma_{i k}(\boldsymbol{\rho})\right)- \\
& -\quad \lambda^{2} \iint d^{2} \boldsymbol{\rho} d^{2} \boldsymbol{\rho}^{\prime} \operatorname{Tr}\left(\sigma_{i k}(\boldsymbol{\rho}) \sigma_{k l}\left(\boldsymbol{\rho}^{\prime}\right)\right) F^{2}\left(\left|\boldsymbol{\rho}-\boldsymbol{\rho}^{\prime}\right|\right)+ \\
& +\imath \int_{0}^{N} d n \dot{\boldsymbol{\rho}}_{i}(n) \dot{\boldsymbol{\rho}}_{k}(n) \psi_{i k}(\boldsymbol{\rho}(n))-\imath \int d^{2} \boldsymbol{\rho} \psi_{i k}(\boldsymbol{\rho}) \sigma_{i k}(\boldsymbol{\rho})
\end{aligned}
$$

and

$$
\Xi(N)=\int \ldots \int \mathcal{D} \boldsymbol{\rho}(n) \mathcal{D} \sigma_{i k}(\boldsymbol{\rho}) \mathcal{D} \psi_{i k}(\boldsymbol{\rho}) \exp \left(-\beta \hat{\mathcal{H}}\left(\dot{\boldsymbol{\rho}}(n), \ddot{\boldsymbol{\rho}}(n), \sigma_{i k}(\boldsymbol{\rho}), \psi_{i k}(\boldsymbol{\rho})\right)\right) .
$$

The above partition function can not be evaluated explicitely because $\psi_{i k}(\boldsymbol{\rho}(n))$ depends on the polymer coordinates and the functional integrals with respect to $\boldsymbol{\rho}(n)$ and $\psi_{i k}(\boldsymbol{\rho}(n))$ can not be separated. However, as recognized by Gupta and Edwards [12] another approximation suggests itself. Replacing the fields $\sigma_{i k}(\boldsymbol{\rho})$ and $\psi_{i k}(\boldsymbol{\rho})$ by their average values $\bar{\sigma}_{i k}$ and $\bar{\psi}_{i k}$, independent of $\boldsymbol{\rho}$ ( and at the same time making the transformation $\imath \bar{\psi}_{i k} \longrightarrow \bar{\psi}_{i k}$ ), one can first of all explicitely evaluate the $\boldsymbol{\rho}(n)$ part of the functional integral

$$
\begin{aligned}
& \int \ldots \int \mathcal{D} \boldsymbol{\rho}(n) e^{-\frac{1}{2} \beta \epsilon(1+F(0))} \int_{0}^{N} \ddot{\boldsymbol{\rho}}(n)^{2} d n-\lambda \int_{0}^{N} \dot{\boldsymbol{\rho}}(n)^{2} d n-\int_{0}^{N} d n \dot{\boldsymbol{\rho}}_{i}(n) \dot{\boldsymbol{\rho}}_{k}(n) \bar{\psi}_{i k}= \\
& =\quad \int \ldots \int \mathcal{D} \boldsymbol{\rho}(n) e^{-2 \pi \sum_{\jmath=-\infty}^{\jmath=+\infty} \boldsymbol{\rho}_{i}(\jmath) \mathcal{M}_{i k}(\jmath) \boldsymbol{\rho}_{k}(-\jmath)}=e^{-\frac{1}{2} \sum_{\jmath=-\infty}^{\jmath=+\infty} \ln \operatorname{Det} \mathcal{M}_{i k}(\jmath)}
\end{aligned}
$$

where

$$
\mathcal{M}_{i k}(\jmath)=\left(\frac{1}{2} \beta \epsilon(1+F(0))\left(\frac{2 \pi_{\jmath}}{N}\right)^{4}+\lambda\left(\frac{2 \pi_{\jmath}}{N}\right)^{2}\right) \delta_{i k}+\left(\frac{2 \pi \jmath}{N}\right)^{2} \bar{\psi}_{i k} .
$$

Above I introduced the Rouse modes of the polymer chain as

$$
\boldsymbol{\rho}(n)=\sum_{\jmath=-\infty}^{\jmath=+\infty} \boldsymbol{\rho}(\jmath) e^{2 \pi \imath \jmath \frac{n}{N}}
$$

The sum of $\ln \operatorname{Det}_{\mathcal{M}_{i k}}(\jmath)$ can be evaluated explicitely and in the limit of long chains where the sum over $\jmath$ can be replaced with an appropriate integration one obtains 12

$$
\frac{1}{2} \sum_{\jmath=-\infty}^{\jmath=+\infty} \ln \operatorname{Det}_{i k}(\jmath)=\frac{N}{2} \sum_{\alpha} \sqrt{\frac{\left(\bar{\psi}_{\alpha}+\lambda\right)}{\frac{\beta \epsilon}{2}(1+F(0))}},
$$


where $\alpha$ is the index of the eigenvalue of $\bar{\psi}_{i k}$. I have omitted a divergent contribution to the above expression since it is irrelevant for subsequent developements. In the limit of 'mean fields' one can now write the free energy corresponding to the partition function Eq.29 as

$$
\beta \mathcal{F}_{M F}=\frac{N}{2} \sum_{\alpha} \sqrt{\frac{\left(\bar{\psi}_{\alpha}+\lambda\right)}{\frac{\beta \epsilon}{2}(1+F(0))}}-N \lambda-\mathcal{S} \sum_{\alpha} \bar{\sigma}_{\alpha} \bar{\psi}_{\alpha}+\lambda F(0) \mathcal{S} \sum_{\alpha} \bar{\sigma}_{\alpha}-\lambda^{2} \mathcal{S} a_{2} \sum_{\alpha} \bar{\sigma}_{\alpha}^{2}+\ldots
$$

where $\mathcal{S}=\int d^{2} \boldsymbol{\rho}$ is the projected area of the surface and I introduced the second virial coefficient $a_{2}$ as

$$
a_{2}=\int d^{2} \boldsymbol{\rho} F^{2}(|\boldsymbol{\rho}|)
$$

Since the mean - field free energy is a function of $\beta \mathcal{F}_{M F}=\beta \mathcal{F}_{M F}\left(\lambda, \bar{\sigma}_{\alpha}, \bar{\psi}_{\alpha}\right)$ I get the equilibrium solution by minimizing it with respect to all three variables:

$$
\begin{aligned}
& \frac{\partial\left(\beta \mathcal{F}_{M F}\right)}{\partial \bar{\psi}_{\alpha}}=0 \Rightarrow \bar{\sigma}_{\alpha}=\frac{\frac{N}{\mathcal{S}}}{4 \sqrt{\frac{\beta \epsilon}{2}(1+F(0))}} \frac{1}{\sqrt{\bar{\psi}_{\alpha}+\lambda}} \\
& \frac{\partial\left(\beta \mathcal{F}_{M F}\right)}{\partial \bar{\sigma}_{\alpha}}=0 \Rightarrow \bar{\psi}_{\alpha}=\lambda F(0)-2 \lambda^{2} a_{2} \bar{\sigma}_{\alpha}+\mathcal{O}\left(\bar{\sigma}_{\alpha}^{2}\right) \\
& \frac{\partial\left(\beta \mathcal{F}_{M F}\right)}{\partial \lambda}=0 \Rightarrow \sum_{\alpha} \frac{\frac{N}{\mathcal{S}}}{4 \sqrt{\frac{\beta \epsilon}{2}(1+F(0))}} \frac{1}{\sqrt{\bar{\psi}_{\alpha}+\lambda}}=\frac{N}{\mathcal{S}}-F(0) \sum_{\alpha} \bar{\sigma}_{\alpha}+\mathcal{O}\left(\bar{\sigma}_{\alpha}^{2}\right) .
\end{aligned}
$$

In order to be consistent the above equations should be linear in the field $\bar{\sigma}_{\alpha}$.

These equations look very much like the analogous equations obtained by Gupta and Edwards [12] for athermal flexible polymer chains with orientation dependent attraction. Solving these equations explicitely for the three fields I obtain the following results. First of all $\bar{\sigma}_{\alpha}$ is a solution of

$$
\left(\bar{\sigma}_{x}-\bar{\sigma}_{y}\right)\left(2 a_{2} \lambda^{2}-\frac{\left(\frac{N}{\mathcal{S}}\right)^{2}}{8(\beta \epsilon)(1+F(0))} \frac{\left(\bar{\sigma}_{x}+\bar{\sigma}_{y}\right)}{\bar{\sigma}_{x}^{2} \bar{\sigma}_{y}^{2}}\right) \text {. }
$$

From the last equation I obtain

$$
\sum_{\alpha} \bar{\sigma}_{\alpha}=\frac{\frac{N}{\mathcal{S}}}{(1+F(0))}
$$

which is simply a consequence of the continuity of the chain since

$$
\begin{aligned}
\operatorname{Tr} \bar{\sigma}_{\alpha} & =\frac{N}{\mathcal{S}}\left\langle\dot{\boldsymbol{\rho}}^{2}(n)\right\rangle=\frac{N}{\mathcal{S}}\left(1-\left\langle\dot{z}^{2}(n)\right\rangle\right)= \\
& =\frac{N}{\mathcal{S}}\left(1-\left\langle\frac{\partial \zeta(\boldsymbol{\rho})}{\partial \boldsymbol{\rho}_{i}} \frac{\partial \zeta(\boldsymbol{\rho})}{\partial \boldsymbol{\rho}_{k}} \dot{\boldsymbol{\rho}}_{i}(n) \dot{\boldsymbol{\rho}}_{k}(n)\right\rangle\right)=\frac{N}{\mathcal{S}}\left(1-F(0)\left\langle\dot{\boldsymbol{\rho}}^{2}(n)\right\rangle\right) .
\end{aligned}
$$


These are the two equations that have to be solved consistently in order to get the dependence of the eigenvalues of the orientation tensor on the surface monomer density, $\frac{N}{\mathcal{S}}$. There is also a boundary condition that we have to take into account, viz. that in the case of a stiff surface $F \longrightarrow 0$ the polymer statistics should reduce to the case of a semiflexible chain embedded in a 2-D surface.

The appropriate solution for $\lambda$ can now be derived from the first two equations of Eqs.36 in the form

$$
\lambda=\frac{(1+F(0))^{2}}{2\left(\beta a_{2}\right) \frac{N}{\mathcal{S}}}\left[1-\sqrt{1-\frac{\left(\beta a_{2}\right)\left(\frac{N}{\mathcal{S}}\right)^{3}}{4 \epsilon(1+F(0))^{4}} \sum_{\alpha} \frac{1}{\bar{\sigma}_{\alpha}^{2}}}\right] .
$$

The nonisotropic (i.e. $\bar{\sigma}_{x} \neq \bar{\sigma}_{y}$ ) solution for the orientational tensor can be obtained from

$$
\frac{\bar{\sigma}_{x}^{2} \bar{\sigma}_{y}^{2}}{\left(\bar{\sigma}_{x}+\bar{\sigma}_{y}\right)}=\frac{\left(\frac{N}{\mathcal{S}}\right)^{2}}{16(\beta \epsilon)(1+F(0)) a_{2} \lambda^{2}} .
$$

Defining the orientational order parameter $S$ as

$$
\bar{\sigma}_{x, y}=\frac{\left(\frac{N}{\mathcal{S}}\right)}{(1+F(0))}(1 \pm S)
$$

so that $\operatorname{Tr} \bar{\sigma}_{\alpha}$ satisfies the constraint Eq. 38, one can derive an approximate equation for $S$ that has two solutions. One corresponding to the isotropic state $(S=0)$ and the other one corresponding to the orientationally ordered phase of polymers $(S \neq 0)$,

$$
S \cong \begin{cases}0 & ; \frac{(1+F(0))^{2}}{(\beta \epsilon) \lambda^{2} a_{2} \frac{N}{\mathcal{S}}}>1 \\ \sqrt{1-\sqrt{\frac{(1+F(0))^{2}}{(\beta \epsilon) \lambda^{2} a_{2}\left(\frac{N}{\mathcal{S}}\right)}}} & ; \frac{(1+F(0))^{2}}{(\beta \epsilon) \lambda^{2} a_{2} \frac{N}{\mathcal{S}}}<1\end{cases}
$$

The above approximate solution is valid only in the vicinity of the critical point $S \cong 0$, where the general solution for $\lambda$ in terms of $S$ can be approximated with its lowest order term. Away from the critical point additional terms in the expansion of $\lambda=\lambda(S)$ would have to be taken into account. The discussion in this contribution will not be extended to this regime of orientational order parameter.

\section{Discussion}

As already stated the existence of effective attraction between segments of a polymer chain embedded in a flexible surface at non-zero temperature is not 
completely unexpected. D'Hoker et al. [9] have found an effective (Casimir) attraction between beads embedded in strings and membranes. Though their model applies to a different situation than our own calculation, its consequences stemm from the same basic physical mechanisms.

The effective nematic interaction of the form $\int_{0}^{N} \int_{0}^{N} d n d n^{\prime} \dot{\boldsymbol{\rho}}_{i}(n) \dot{\boldsymbol{\rho}}_{k}(n)$ $\dot{\boldsymbol{\rho}}_{l}\left(n^{\prime}\right) \dot{\boldsymbol{\rho}}_{m}\left(n^{\prime}\right)\left\langle\mathbf{n}_{i}(n) \mathbf{n}_{m}\left(n^{\prime}\right)\right\rangle\left\langle\mathbf{n}_{k}\left(n^{\prime}\right) \mathbf{n}_{l}(n)\right\rangle$ can in fact be viewed as a special type of Casimir force between different segments of a polymer chain. It is due to the change in the energy of conformational fluctuations of the supporting surface ('zero point energy' 13] in the case of electromagnetic Casimir forces) in the presence of embedded chains. It is analogous to the zero order van der Waals interaction energy that is due purely to thermodynamic fluctuations [14].

The dependence of this Casimir force on the orientation of the polymer segments comes essentially from the geometric constraint for the polymer chain, i.e. $\dot{\mathbf{r}}^{2}(n)=1$, since some of the 3 -D configurations are excluded if the chain is confined to lye in the embedding 2-D surface. Its spatial dependence on the other hand, thus the form of $F(|\rho|)$, stemms purely from the nature of the bare surface fluctuations.

Once the presence of a nematic Casimir interaction is derived it naturally leads to an orientational ordering transition. Also the order of the transition clearly follows from the fact that the effective polymer partition function corresponds to a polymer confined to a 2-D surface. Nematic transition on a 2-D surface is of a second order [15]. Whether the order of the transition is preserved for non-ideal polymers and/or higher orders in the perturbation expansion remains to be assesed.

The present calculation is of importance for phenomena involving interface embedded polymers. Monomolecular films of poly(dimethyl siloxane) [2] show domains of differing surface density even below the submonolayer concentrations. Orientational ordering described in this contribution could possibly lead to domains of different surface density of polymers at quite small average surface densitites. One way of testing this mechanism is to quench the free surface fluctuations and thus attenuate the nematic Casimir attraction which should prevent the ordering of the embedded polymers.

Some of the open questions that we leave for further discussion is whether the nature of the transition is conserved also in higher orders of the perturbation expansion in terms of $F$ and what is the interplay between the repulsive (steric) interactions and the nematic Casimir attraction. 


\section{References}

[1] Ringsdorf, H., Schlarb, B., Venzmer, J. Angew.Chemie 100 (1988) 117.

[2] Mann, E.K., Hénon, S., Langevin, D., Meunier, J., J.Phys. II France 2 (1992) 1683.

[3] Dvolaitzky, M., Guedeau - Boudeville, M.A., Leger, L., Langmuir 8 (1992) 2595.

[4] Kozlov, M.M., Helfrich, W., Langmuir 10 (1994) 4219 (and references therein).

[5] Kozlov, M.M., Helfrich, W., Langmuir 9 (1993) 2761.

Kozlov, M.M., Helfrich, W., J.Phys. II France 4 (1994) 1427.

Kozlov, M.M., Helfrich, W., Phys.Rev. E 51 (1995) (in print).

[6] Li, H., Kardar, M., Phys.Rev. A 46 (1992) 6490.

[7] Kardar, M., Orland, H., (1995) (cond-mat preprint)

[8] Dalley, S., (1995) (cond-mat preprint)

[9] D’Hoker, E., Sikivie, P., Kanev, Y., (1995) (cond-mat preprint)

[10] Bawendi, M.G., Freed, K.F., J.Chem.Phys. 83 (1985) 2491.

Lagowski, J.B., Noolandi, J., J.Chem.Phys. 95(1991) 1266.

[11] Doi, M. and Edwards, S.F., The Theory of Polymer Dynamics (Clarendon Press, Oxford, 1986).

[12] Gupta, A.M., Edwards, S.F., J.Chem.Phys. 98 (1993) 1588.

Gupta, A.M., Edwards, S.F., Polymer 34 (1993) 3112.

[13] Milloni, P.W., The Quantum Vacuum (Academic Press, New York, 1994).

[14] Mahanty, J., Ninham, B.W., Dispersion forces (Academic Press, New York, 1976).

[15] Frenkel, D., Statistical mechanics of liquid crystals in Liquids, Freezing and Glass Transition, Eds. Hansen, J.P., Levesque, D., Zinn-Justin, J. (Elsevier, Amsterdam, 1991). 